\title{
An Experimental Reproduction of Necrotic Enteritis in Broiler Chickens
}

Keywords: Clostridium perfringens; Necrotic enteritis; Broiler; Experimental model

\begin{abstract}
Clostridium perfringens is the main causative agent of avian necrotic enteritis (NE), an enteric infectious disease considered among the most important diseases in the poultry industry. Currently, there are numerous reports of experimental reproduction of NE using different inoculation protocols along with various predisposing factors which produce highly variable results. These models represent a fraction of a wide range of farming conditions under which disease can develop. This work describes the experimental induction of C. perfringens NE in broiler chickens after a short feed withdrawal of 12 hours prior to bacterial challenge. Broiler chicks fed with commercial chick starter for 14 days post-hatch were afterwards offered feed mixed with C. perfringens twice a day for three consecutive days. On average, over $60 \%$ of challenged birds developed typical gross lesions. The results show that it is possible to reproduce the disease under conditions similar to those found in poultry farms which are not covered by other developed models. This model proved to be effective in the experimental reproduction of $\mathrm{NE}$, allowing the evaluation of pathological parameters.
\end{abstract}

\section{Introduction}

Clostridium perfringens is a Gram-positive, rod-shaped, anaerobic, spore-forming bacterium that is commonly found in soil, sewage and in the gastro-intestinal tract of animals and humans as a member of the normal gut microbiota. According to the current classification, $C$. perfringens isolates are divided into five types (A, $\mathrm{B}, \mathrm{C}, \mathrm{D}$ and $\mathrm{E}$ ) on the basis of the production of four major toxins (alpha, beta, epsilon and iota) [1]. Certain strains of $C$. perfringens type A cause necrotic enteritis (NE) in poultry [2,3]. In broilers, $\mathrm{NE}$ appears as a sudden increase in mortality occurring at any time during the production cycle, up to $1 \%$ per day and it will continue for up to two weeks if the affected flock left untreated. The subclinical form of the disease is characterized by non-specific signs such as poor digestion, reduced weight gain and increased feed conversion ratio, without obvious increase in mortality. Typical necrotic lesions can be observed in the intestinal tract during necropsy $[3,4]$. Both presentations of the disease have become economically significant problems for the broiler industry worldwide.

$\mathrm{NE}$ is considered a complex and multi-factorial disease. Experimental attempts to reproduce NEhave required the inclusion of one or more potential predisposing factors. Lesions of NE associated with the challenge of birds with $C$. perfringens alone have been reported in a relatively low proportion of animals [5]. Recently, an effective model of NE which reproduced consistently several clinical signs of the disease and intestinal lesions in birds fed with a highprotein diet has been described by Cooper and Songer [6]. Although high protein diet would serve as potential predisposing factor, the successful use of this model seems to depend on the complete
Journal of

Veterinary Science \& Medicine

\author{
L. M. Redondo ${ }^{1,2^{*}}$, E. A. Redondo ${ }^{1,2}$, F. Delgado ${ }^{1}$, L. F. \\ La Sala ${ }^{2,3}$ and M. E. Fernández Miyakawa ${ }^{1,2}$ \\ ${ }^{\text {I} I n s t i t u t o ~ d e ~ P a t o b i o l o g i ́ a, ~ C e n t r o ~ N a c i o n a l ~ d e ~ I n v e s t i g a c i o n e s ~}$ \\ Agropecuarias, Instituto Nacional de Tecnología Agropecuaria, \\ Calle Las Cabañas y Los Reseros s/n, Casilla de Correo 25 (1712), \\ Castelar, Buenos Aires, Argentina \\ ${ }^{2}$ Consejo Nacional de Investigaciones Científicas y Técnicas, \\ Rivadavia 1917 (1033) Ciudad Autónoma de Buenos Aires, Argentina \\ ${ }^{3}$ GEKKO, Grupo de Estudios en Conservación y Manejo, \\ Departamento de Biología, Bioquímica y Farmacia, Universidad \\ Nacional del Sur, San Juan 670, 8000 Bahía Blanca, Buenos Aires, \\ Argentina \\ *Address for Correspondence \\ L. M. Redondo, Instituto de Patobiología, Centro Nacional de \\ Investigaciones Agropecuarias, Instituto Nacional de Tecnología \\ Agropecuaria, Calle Las Cabañas y Los Reseros s/n, Casilla de Correo \\ 25 (1712), Castelar, Buenos Aires, Argentina, Fax: 0054-11-46210443; \\ E-mail: redondo.leandro@inta.gob.ar
}

Submission: 20 January, 2016

Accepted: 11 March, 2016

Published: 15 March, 2016

Copyright: ( $\odot 2016$ Redondo LM, et al. This is an open access article distributed under the Creative Commons Attribution License, which permits unrestricted use, distribution, and reproduction in any medium, provided the original work is properly cited.

Reviewed \& Approved by: Dr. Huaguang Lu, Department of Animal Science and Veterinary Medicine, Penn State University, USA

preservation of virulent characteristics of the pathogenic strain used. Therefore, the goal of the present report was to determine if the use of an optimized culture of a freshly pathogenic C. perfringens strain can cause a consistent reproduction of the disease in broiler chicks fed with regular feed but starved for 12 hours as predisposing condition.

\section{Materials and Methods}

\section{Chickens, facilities and experiment design}

Cobb Broiler chicks were obtained as one-day old hatchlings from a commercial hatchery (Buenos Aires, Argentina). Birds were housed in biosafety level 2 facilities located in the Veterinary and Agriculture Research Center (CICVyA-INTA), with controlled temperature and humidity and automated ventilation system. Eighty chicks were divided into 4 groups, 20 birds per group. Each group was housed in $1.5 \mathrm{~m}^{2}$ and $80 \mathrm{~cm}$ high pens made of wire mesh $(0.5 \mathrm{~mm}$ thick $)$ and sheets of flooring hardboard. Wood shavings beddings were used at the time of starting the trial and maintained during each experiment. Commercial starter rations $(3200 \mathrm{Kcal} / \mathrm{kg} \mathrm{EM}, 20 \%$ total protein; Alimcer S.A., Buenos Aires, Argentina) were given ad-libitum in galvanized feed trays (regular diet). Commercial feed was mixed with fishmeal in a 50:50 proportion to obtain a high protein diet (HPD) to reproduce conditions favoring $C$. perfringens lesions used in previous assays [6]. Birds used in these studies were unvaccinated and fed with ration free of antimicrobial growth promoters.

\section{Clostridium perfringens strain and inoculum preparation}

The C. perfringens strain used in these studies was isolated from the intestinal lesions of a three weeks old broiler chicken during 
an outbreak of NE in a commercial poultry farm of Buenos Aires, Argentina in 2012. Molecular toxinotyping shows that this isolate was a type A strain negative for NetB [7] and $c p b 2$ [6]. For inoculums preparation, a glycerol aliquot of the mentioned strain, obtained immediately after the first isolation, was streaked onto a blood agar plate with $5 \%$ defibrinated bovine blood. After incubation in anaerobic atmosphere $(5 \% \mathrm{H} 2: 5 \% \quad \mathrm{CO} 2: 90 \% \mathrm{~N} 2)$ at $37{ }^{\circ} \mathrm{C}$ for 18 hours, $1-2$ colonies were transferred into $10 \mathrm{ml}$ cooked meat medium (CMM; Difco) and incubated in anaerobiosis at $37^{\circ} \mathrm{C}$ for 12 hours. Then, this culture was inoculated into $100 \mathrm{ml}$ of thioglycollate broth (FTG) and cultured as before. The mentioned FTG culture was diluted 1:10 in sterile CMM and incubated in anaerobiosis at $37^{\circ} \mathrm{C}$ for 12 hours. One hundred $\mathrm{ml}$ of the last CMM culture was used to inoculate $1 \mathrm{~L}$ of FTG, and incubated during 12 hours. After incubation, a drop plate method [8] was used to assess the number of colony-forming units (CFU) in each dose of the inoculums. Final culture was mixed with feed in a 1:1 (w/v) ratio and administered immediately to birds. The procedure was repeated for each dose of inoculum used during the challenge (total challenge feeding, $\mathrm{n}=6$ ).

\section{Challenge protocol}

A $2 \times 2$ arrangement of treatments was used to test the effect of C. perfringens challenge (unchallenged vs. challenged) and diet effect (regular vs. high protein). As mentioned above, four groups of 20 birds were assigned to each treatment. On day 15 and before first challenge birds were fasted for 12 hours. Between days 16 to 18 , birds were challenged twice a day with final $C$. perfringens culture mixed with feed. Uneaten feed was discarded before each subsequent feeding. Sterile FTG mixed with feed was administered to a group of birds fed with regular diet and to a group of birds with HPD as negative control. This protocol was applied equally to birds fed with regular rations and high protein ration. This challenge was repeated three times and results were expressed as the mean of the three different trials.

\section{Clinical and pathological examination}

After first dose of inoculum, birds were observed three times a day for the prompt detection of clinical signs. On day 19 (three days after first challenge) birds were euthanized and necropsy was performed immediately for examination of gross lesions. Intestinal tracts were removed and lesions were scored blindly by two experienced pathologists (scores: $0=$ no apparent gross lesions; $1=$ removable fibrin deposit; $2=$ isolated focal necrosis or ulceration ( 1 to 5 foci); $3=$ =multiple focal necrosis or ulceration (6 or more foci); $4=$ extensive areas of necrosis; $5=$ diffuse necrosis, presence of attached pseudomembrane). To confirm the identity of intestinal lesions, tissue samples were taken from each bird with gross lesions. Samples were kept refrigerated or in buffered formalin solution for both bacteriological and histopathological diagnosis. Macroscopic characteristics and weight of livers were recorded for each bird.

\section{Histopathology}

Intestinal segments showing gross lesions compatible with $\mathrm{NE}$ were analyzed microscopically. Samples from duodenum, jejunum and ileum were fixed in $10 \%$ phosphate-buffered formalin, paraffinembedded, sectioned, stained, and examined for microscopic lesions.

\section{Bacteriology}

Intestinal content samples were inoculated directly onto agar McConkey plates, brain and heart infusion (BHI) and tetrathionate broth (as a pre-enrichment step), and then incubated at $37^{\circ} \mathrm{C}$ for 24 hours. For anaerobes diagnosis, samples were processed similarly by inoculating bovine blood agar plates with neomycin $(100 \mu \mathrm{g} / \mathrm{ml})$ [9] and pre-enriching in cooked meat medium. Inoculated media were incubated in anaerobic jar (Oxoid) in atmosphere composed of $\mathrm{H}_{2} 10 \%: \mathrm{CO}_{2} 10 \%: \mathrm{N}_{2} 80 \%$ for 18 hours. Colonies displaying $C$. perfringens characteristics were isolated, cultured in CMM and incubated at $37^{\circ} \mathrm{C}$ for 18 to 24 hours.

\section{Growth performance and feed conversion rate}

In order to evaluate the impact of $C$. perfringens challenge on growth and feed conversion rate, trials were repeated and the observation time was extended to15 days after the first dose of inoculums. In this set of experiments, two groups of 20 birds (unchallenged vs. challenged) were maintained and challenged as described above. According with previous results no fishmeal supplementation was included in these trials. Animals were clinically examined three times a day and weighed twice a week. Total feed intake per pen was also measured. At the end of the trial body weight and feed conversion rate were calculated per pen for each period and for the overall period [10].

\section{Statistical analysis}

The associations between the variables were studied using generalized linear models (GLM) with a binary, logistic response. All the analyses were performed using the statistical package $R$ ( $R$ Development Core Team, 2012). GLMs were fitted using the R base 'stats' package and linear models were fitted using the generalized least squares method using the R 'nlme' package. A two-tailed Fisher exact test was used for statistical comparison of rates of lesion development across groups. An unpaired t-test was used to examine differences in average lesion scores across groups [6].

\section{Institutional animal care and use committee (IACUC) approval}

Animal trials described in the present work were reviewed and approved by the CICVyA-INTAIACUC, under protocol number

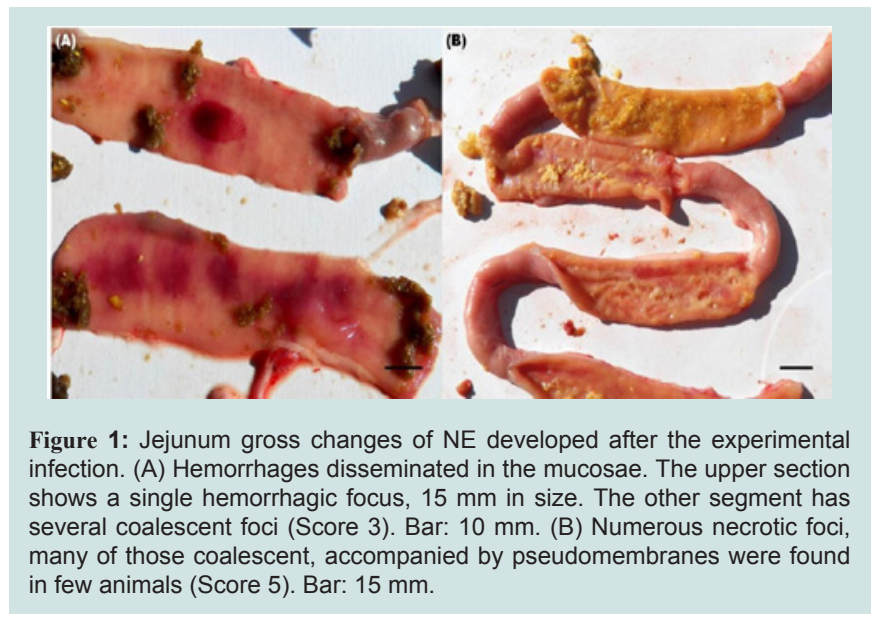




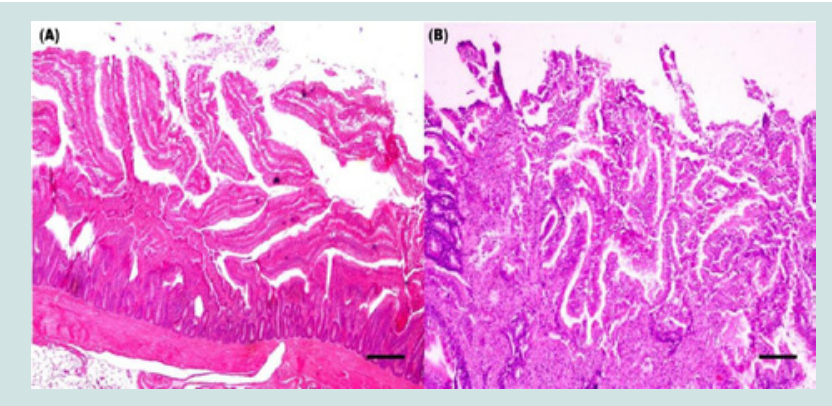

Figure 2: Jejunum microscopic changes of NE developed after the experimental infection. (A) Necrosis of cells from the apex of villi, with congestion and small hemorrhages in the propria, corresponding with a gross lesion score 2. Bar: 1000um. (B) Diffuse necrosis of villi, with thickness of the lamina propria by congestion and infiltration of inflammatory cells, corresponding with a gross lesion score 4. Bar: 250 um.

\section{0/2010.}

\section{Results}

\section{High protein diet vs. regular diet}

Initial attempts to reproduce NE lesions in broiler chickens without feed withheld produced lesions in less than $40 \%$ of the challenged birds consuming regular or high protein diets (data not shown). In subsequent experiments, birds were fasted for 12 hours before first dose of $C$. perfringens inoculum. In the high protein diet group, gross lesions (Figure 1) were observed in $62 \%$ of the challenged birds which was statistically different to the corresponding control group $(12 \% ; \mathrm{P}<0.05)$. In the regular diet group, $63 \%$ of the challenged birds developed gross lesions which was statistically different to the corresponding control group $(0 \% ; \mathrm{P}<0.05)$. Differences in the average gross lesion score between challenged and unchallenged groups were only significant in the birds fed with regular diet. In birds fed with HPD, intestinal gross lesions were more severe in the challenged group ( 1.80 vs. $0.90 ; \mathrm{P}>0.05$ ). In birds fed with a regular diet, gross lesions were as well more severe in the challenged group (2.70 vs.
0.00; $\mathrm{P}<0.01$ ). Based on our data, we were unable to demonstrate statistically that chickens fed with a HPD in addition to challenge with $C$. perfringens have increased odds of pathology compared with challenged birds under a regular protein diet. The association between "pathology" (present/absent) and exposure was assessed using a bivariate GLM. Results from experiments with chickens under HPD or regular diets challenged with C. perfringens are summarized in Table 1.

\section{Necropsy and histopathology findings}

Challenged birds necropsied three days after the initial challenge showed lesions in the jejunum and ileum (eventually in cecum) being more frequent in this last one $(72 \%$ vs. $43 \%$, considering all the independent assays performed with 60 birds) and with higher odds of injury in jejunum $(\mathrm{OR}=9.05,95 \% \mathrm{CI}=3.3-29.3, \mathrm{P}<0.0001)$ than ileum $(\mathrm{OR}=3.7,95 \% \mathrm{CI}=1.5-10.3, \mathrm{P}=0.00741)$. In total, lesions were observed in $70 \%$ of the inoculated birds whereas none of the chicks in the control group developed gross lesions compatible with NE. Lesion score distribution for each portion of the small intestine are summarized in Tables 2 and 3.

Microscopic observation confirms the identity of recorded gross lesions. Microscopic lesions observed in tissue sections from the inoculated group included foci of necrosis, haemorrhage and epithelium desquamation. In more severe cases, accumulation of fibrinous exudate was observed (Figure 2). Mucosal smears of the small intestine of the inoculated group showed abundant short Gram positive bacilli compatible with $C$. perfringens. Although intestinal gross lesions were observed in the control group, microscopic changes were not compatible with NE lesions.

Abundant and pure growth of anaerobic bacilli compatible with C. perfringens was obtained from intestinal gross lesions samples of the inoculated group. The identity of the suspected colonies, randomly sampled, was confirmed by molecular and biochemical methods. C. perfringens colonies were not obtained from samples of the control group.

Table: 1 Gross intestinal lesion frequency and scores of chickens experimentally challenged with C. perfringens.

\begin{tabular}{|c|c|c|c|c|c|c|}
\hline Diet (treatment) & $\begin{array}{l}\text { C. perfringens } \\
\text { challenge }\end{array}$ & $\begin{array}{l}\text { Odds } \\
\text { Ratio }\end{array}$ & $\begin{array}{l}\text { Birds with gross lesions/ total } \\
\text { birds (\%) }\end{array}$ & $\begin{array}{l}\text { Average lesion } \\
\text { score }\end{array}$ & $\begin{array}{l}\text { Birds with lesions in } \\
\text { jejunum (\%) }\end{array}$ & $\begin{array}{l}\text { Birds with } \\
\text { lesions in } \\
\text { ileum }(\%)\end{array}$ \\
\hline \multirow{2}{*}{ High protein diet } & Inoculated & \multirow{2}{*}{3} & $10 / 16(62 \%)^{* 1}$ & $1,8 \pm 0.82^{* 1}$ & $10 / 10(100 \%)$ & $5 / 10(50 \%)$ \\
\hline & Control & & $2 / 16(12 \%)^{* 1}$ & $0,9 \pm 0.67^{* 1}$ & $1 / 2(50 \%)$ & $1 / 2(50 \%)$ \\
\hline \multirow{2}{*}{ Regular diet } & Inoculated & \multirow[b]{2}{*}{5,3} & $17 / 27(63 \%)^{* 2}$ & $2,7 \pm 0.5^{\star 2}$ & $17 / 17(100 \%)$ & $13 / 17(76 \%)$ \\
\hline & Control & & $0 / 25(0 \%)^{* 2}$ & $0^{* 2}$ & N/A & N/A \\
\hline
\end{tabular}

$\star_{1,2}$ Values with matching superscripts have statistically significant differences in percent of birds developing lesions $(p<0.05)$. 


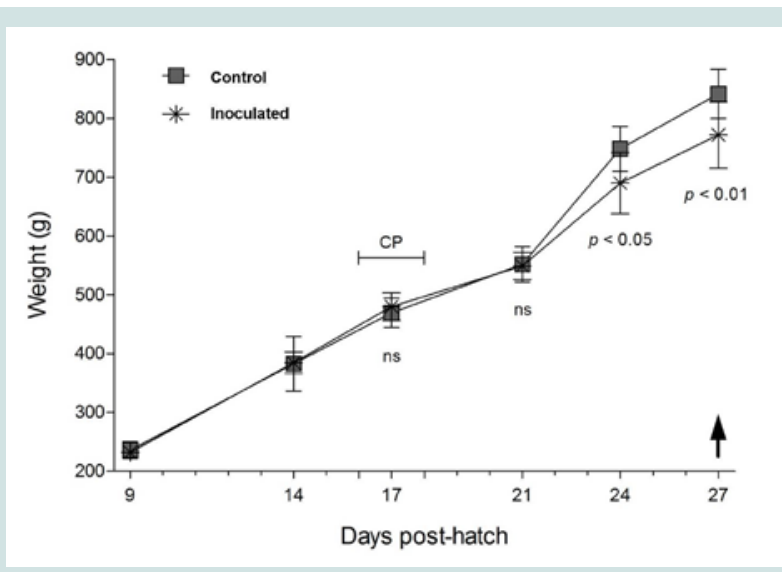

Figure 3: Mean live weight (symbols) and $95 \% \mathrm{Cl}$ (whiskers) in the contro $(n=20)$ and inoculated $(n=17)$ groups. $C P=C$. perfringens challenge. Arrow indicates termination point of the trial. The difference in weight between groups was analyzed statistically for the period between 15 (inoculation) and 27 days post-hatch. Levels of significance for the pairwise comparisons are presented below each pair. Ns=Not significant results.

Clinical signs consistent with NE in any of its presentations were not observed during the challenge although diarrhoea and also blood-streaked stool was commonly found in beddings and some of the birds in the inoculated groups showed signs of depression and reluctance to move after the challenge. In the control groups, no bird showed such changes at any time. No morphological alterations were observed in the liver of the inoculated birds; neither significant association was observed in any of the four linear regression models tested, each including liver weight (continuous) as dependent variable and: 1) global lesion score, 2) total lesion score, 3) jejunum score, and 4) ileum score, as independent variables in each of these models.

\section{Weight gain and feed conversion}

The effects of NE over bodyweight gain and food consumption was registered throughout a modified version of the trial. Because three days post challenge were not enough to observe change sat the production level, the trail was extended up to 15 days post challenge. At the end of this test, the control group achieved a greater average body weight gain compared to C. Perfringens inoculated group (841.8 grs vs. 771.9 grs, see Figure 3). In the challenged group, an inverse correlation between weight gain and degree of injury was observed, particularly in birds with lesions of score 3 or higher, although it was not statistically significant. No differences were observed in total feed consumption between inoculated and control groups, although, feed efficiency was lower within the first group (1.76 vs. 1.71).

\section{Discussion}

NE is a complex, multifactorial disease with many unknown factors influencing its occurrence and the severity of outbreaks. Nearly all developed models of NE depend on the presence of predisposing factors, two of the most important being mucosal damage caused by coccidian pathogens $[11,12]$ and feed containing high protein levels [6]. The experiments performed in the present report show a successful and consistent experimental reproduction of NE lesions in birds challenged during three days with $C$. perfringens cultures, with no significant differences either if high protein levels were or not added in the feed. These results show that NE can be reproduced by the sole administration of pathogenic $C$. perfringens with no other predisposing factor than feed withdrawal.

It has been described that $C$. perfringens strains derived from clinically healthy broilers or other animal species did not produce NE in broilers, even administering high numbers of $C$. perfringens cells in the gut and despite the use of predisposing factors [13]. Recent experiments show that only certain $C$. perfringens strains are capable to induce NE in chickens and that those strains normally constitute only a minority in the intestinal tract of healthy chickens. Cooper and Songer suggested that the potential of the strains isolated from field cases of NE to reproduce lesions diminishes with in vitro passages [6]. Also, we have observed a lower pathogenicity of NE strains after repeated in vitro subculturing (data not shown). Concordantly, previous works report that these C. perfringens NE isolates emerge as very specialized strains $[13,14]$, which require determined virulence factors codified in plasmids or other mobile genetic elements to be fully pathogenic [15]. Therefore, the use of fresh or well preserved isolates of $C$. perfringens from cases of NE seems to be determinant to induce lesions during the experimental reproduction of NE in broilers. Also, an optimized culture protocol seems to be necessary for an effective C. perfringens challenge as Cooper and Songer stated that simplified

Table 2: Score distribution of jejunum gross lesions.

\begin{tabular}{|c|c|c|c|c|}
\hline \multirow{2}{*}{$\begin{array}{c}\text { Gross lesion } \\
\text { score }\end{array}$} & \multicolumn{2}{|c|}{$\begin{array}{c}\text { Birds fed with } \\
\text { regular diet }\end{array}$} & \multicolumn{2}{c|}{$\begin{array}{c}\text { Birds fed with } \\
\text { high protein diet }{ }^{1}\end{array}$} \\
\cline { 2 - 5 } & Inoculated & Control & Inoculated & Control \\
\hline $0^{2}$ & $0 / 17$ & 0 & $0 / 10$ & $0 / 2$ \\
\hline 1 & $3 / 17$ & 0 & $2 / 10$ & $1 / 2$ \\
\hline 2 & $4 / 17$ & 0 & $4 / 10$ & $0 / 2$ \\
\hline 3 & $5 / 17$ & 0 & $2 / 10$ & $0 / 2$ \\
\hline 4 & $5 / 17$ & 0 & $2 / 10$ & $0 / 2$ \\
\hline$n$ & $17 / 17$ & 0 & $10 / 10$ & $1 / 2$ \\
\hline
\end{tabular}

${ }^{1}$ Birds with jejunum NE compatible gross lesions/ Birds with intestinal NE compatible gross lesions.

${ }^{2}$ Gross lesion score $=0$ includes birds without evident NE compatible gross lesions.

Table 3: Score distribution of ileum gross lesions.

\begin{tabular}{|c|c|c|c|c|}
\hline \multirow{2}{*}{$\begin{array}{c}\text { Gross lesion } \\
\text { score }\end{array}$} & \multicolumn{2}{|c|}{ Birds fed with regular diet ${ }^{1}$} & \multicolumn{2}{|c|}{$\begin{array}{c}\text { Birds fed with high protein } \\
\text { diet }^{1}\end{array}$} \\
\cline { 2 - 5 } & Inoculated & Control & Inoculated & Contro \\
\hline $0^{2}$ & $0 / 17$ & 0 & $0 / 10$ & $0 / 2$ \\
\hline 1 & $0 / 17$ & 0 & $1 / 10$ & $1 / 2$ \\
\hline 2 & $6 / 17$ & 0 & $2 / 10$ & $0 / 2$ \\
\hline 3 & $4 / 17$ & 0 & $2 / 10$ & $0 / 2$ \\
\hline $4-5$ & $3 / 17$ & 0 & $0 / 10$ & $0 / 2$ \\
\hline$n^{2}$ & $13 / 17$ & 0 & $5 / 10$ & $1 / 2$ \\
\hline
\end{tabular}

${ }^{1}$ Birds with ileum NE compatible gross lesions/ Birds with intestinal NE compatible gross lesions.

${ }^{2}$ Gross lesion score $=0$ includes birds without evident NE compatible gross lesions. 
Citation: Redondo LM, Redondo EA, Delgado F, Sala LF, Miyakawa ME. An Experimental Reproduction of Necrotic Enteritis in Broiler Chickens. J Veter Sci Med. 2016;4(1): 5.

sub-culture methods used for preparing inocula were unsatisfactory at reproducing disease [6]. This optimized culture protocol is one of the main differences with the model used by Olkowski et al. in which gross pathological changes in intestinal tissues were observed in many of the challenged birds but upon histological examination none of the experimentally exposed birds showed evident mucosal necrosis typical of field cases of NE [16].

The model used in the present report was able to reproduce lesions observed in field cases of NE, occurring principally in jejunum, then ileum, and eventually in cecum. Similarly, lesions in field cases of NE are most common in jejunum, followed by ileum, duodenum, and cecum $[4,17]$. For macroscopic evaluation of intestinal lesions we used a score similar to what has been reported in previous works $[6,18,19]$. However, unlike these studies, we did not include the criteria of "thin walled" and "friable" intestine with gas accumulation, because it is difficult to define objectively [20].

The C. perfringens strain used in the present report was selected on basis of the evident intestinal lesions and signs of NE in chickens, resulting in a NetB and $c p b 2$ negative strain by PCR. Although recent works suggest a critical association between NetB producing strains and NE [21], not all NE strains harbor NetB [22] and these NetB-negative $C$. perfringens strains also produce NE [6]. On the other hand, Beta2 toxin may have a role in the pathogenesis of $\mathrm{NE}$ but surveillance of healthy chickens and chickens with NE have not revealed a direct correlation between occurrence of disease and presence of $c p b 2$ in isolates [20]. Therefore, further studies with isolates of diverse genetic background, including NetB toxin positive and negative strains are planned to compare virulence observed in the field and the NE model presented here.

\section{Conclusions}

Experimental conditions used in the present study, without the inclusion of severe changes on feed formulation (high protein levels) or other frequent predisposing factors as cocciddia or immunosupression, allow the consistent reproduction of $\mathrm{NE}$ in broiler chickens. This experimental approach using temporary starving as unique predisposing factor would be useful to evaluate treatments directed against $C$. perfringens. Submitting birds to feed restriction is a common practice in poultry industry intended to reduce economic losses caused by heat stress. Therefore, this model represents infection conditions that are not represented by other models and can occur during commercial breeding.

\section{References}

1. Songer JG (1996) Clostridial enteric diseases of domestic animals. Clin Microbiol Rev 9: 216-234.

2. Al-Sheikhly F, Truscott RB (1977) The pathology of necrotic enteritis of chickens following infusion of Clostridium perfringens into the duodenum. Avian Dis 21: 230-240

3. Kaldhusdal M, Schneitz C, Hofshagen M, Skjerve E (2001) Reduced incidence of Clostridium perfringens-associated lesions and improved performance in broiler chickens treated with normal intestinal bacteria from adult fowl. Avian Dis 45: 149-156.

4. Van Immerseel F, De Buck J, Pasmans F, Huyghebaert G, Haesebrouck F, et al. (2004) Clostridium perfringens in poultry: an emerging threat for animal and public health. Avian Pathol 33: 537-549.

5. Collier CT, Hofacre CL, Payne AM, Anderson DB, Kaiser P, et al. (2008) Coccidia-induced mucogenesis promotes the onset of necrotic enteritis by supporting Clostridium perfringens growth. Vet Immunol Immunopathol 15: 104-115.

6. Cooper KK, Songer JG (2010) Virulence of Clostridium perfringens in an experimental model of poultry necrotic enteritis. Vet Microbiol 142: 323-328.

7. Keyburn AL, Boyce JD, Vaz P, Bannam TL, Ford ME, et al. (2008) NetB, a new toxin that is associated with avian necrotic enteritis caused by Clostridium perfringens. PLoS Pathog 4: e26.

8. Miles AA, Misra SS, Irwin JO (1938) The estimation of the bactericidal power of the blood. J Hyg (Lond) 38: 732-749.

9. Marshall RS, Steenbergen JF, Mcclung LS (1965) Rapid technique for the enumeration of Clostridium perfringens. Appl Microbiol 13: 559-563.

10. Murugesan GR, Syed B, Haldar S, Pender C (2015) Phytogenic feed additives as an alternative to antibiotic growth promoters in broiler chickens. Front Vet Sci 2: 21.

11. Al-Sheikhly F, Al-Saieg A (1980) Role of Coccidia in the occurrence of necrotic enteritis of chickens. Avian Dis 24: 324-333.

12. Williams RB, Marshall RN, La Ragione RM, Catchpole J (2003) A new method for the experimental production of necrotic enteritis and its use for studies on the relationships between necrotic enteritis, coccidiosis and anticoccidial vaccination of chickens. Parasitol Res 90: 19-26.

13. Timbermont L, Lanckriet A, Pasmans F, Haesebrouck F, Ducatelle R, et al. (2009) Intra-species growth-inhibition by Clostridium perfringens is a possible virulence trait in necrotic enteritis in broilers. Vet Microbiol 137: 388-391.

14. Chalmers G, Bruce HL, Hunter DB, Parreira VR, Kulkarni RR, et al. (2008) Multilocus sequence typing analysis of Clostridium perfringens isolates from necrotic enteritis outbreaks in broiler chicken populations. J Clin Microbiol 46: 3957-3964

15. Lepp D, Roxas B, Parreira VR, Marri PR, Rosey EL, et al. (2010) Identification of novel pathogenicity loci in Clostridium perfringens strains that cause avian necrotic enteritis. PLoS One 5: e10795.

16. Olkowski AA, Wojnarowicz C, Chirino-Trejo M, Drew MD (2006) Responses of broiler chickens orally challenged with Clostridium perfringens isolated from field cases of necrotic enteritis. Res Vet Sci 81: 99-108.

17. Long JR, Pettit JR, Barnum DA (1974) Necrotic enteritis in broiler chickens. II. Pathology and proposed pathogenesis. Can J Comp Med 38: 467-474.

18. McReynolds JL, Byrd JA, Genovese KJ, Poole TL, Duke SE, et al. (2007) Dietary lactose and its effect on the disease condition of necrotic enteritis. Poult Sci 86: 1656-1661.

19. Keyburn AL, Sheedy SA, Ford ME, Williamson MM, Awad MM, et al. (2006) Alpha-toxin of Clostridium perfringens is not an essential virulence factor in necrotic enteritis in chickens. Infect Immun 74: 6496-6500.

20. Gholamiandehkordi AR, Timbermont L, Lanckriet A, Van Den Broeck W, Pedersen K, et al. (2007) Quantification of gut lesions in a subclinical necrotic enteritis model. Avian Pathol 36: 375-382.

21. Keyburn AL, Yan XX, Bannam TL, Van Immerseel F, Rood Jl, et al. (2010) Association between avian necrotic enteritis and Clostridium perfringens strains expressing NetB toxin. Vet Res 41: 21.

22. Hibberd MC, Neumann AP, Rehberger TG, Siragusa GR (2011) Multilocus sequence typing subtypes of poultry Clostridium perfringens isolates demonstrate disease niche partitioning. J Clin Microbiol 49: 1556-1567.

\section{Acknowledgements}

This research was supported by Instituto Nacional de Tecnología Agropecuaria Grant INTA. AESA-203941. We also thank the Consejo Nacional de Investigaciones Científicas y TecnológicasArgentina for the support. We thank DVM Javier Quintar and DVM Claudio Cabral for their assistance and Mr. Jose Vallejos, Mr. Ignacio de la Fuente, Mr. Pablo Huertas and Ms. Laura Gonzales, for invaluable help at the lab. We thank Ms. Daniela Losada Eaton for her manuscript correction. 